\title{
Exploring Mixed Reality Robot Communication Under Different types of Mental Workload
}

\author{
Nhan Tran \\ Colorado School of Mines \\ Department of Computer Science \\ nttran@mines.edu \\ Thao Phung \\ Colorado School of Mines \\ Department of Computer Science \\ thaophung@mines.edu
}

\author{
Kai Mizuno \\ Colorado School of Mines \\ Department of Computer Science \\ kmizuno@mines.edu \\ Leanne Hirshfield \\ University of Colorado \\ Department of Computer Science \\ leanne.hirshfield@colorado.edu
}

\author{
Trevor Grant \\ University of Colorado \\ Department of Computer Science \\ trevor.grant@colorado.edu \\ Tom Williams \\ Colorado School of Mines \\ Department of Computer Science \\ twilliams@mines.edu
}

\begin{abstract}
This paper explores the tradeoffs between different types of mixed reality robotic communication under different levels of user workload. We present the results of a within-subjects experiment in which we systematically and jointly vary robot communication style alongside level and type of cognitive load, and measure subsequent impacts on accuracy, reaction time, and perceived workload and effectiveness. Our preliminary results suggest that although humans may not notice differences, the manner of load a user is under and the type of communication style used by a robot they interact with do in fact interact to determine their task effectiveness.
\end{abstract}

\section{KEYWORDS}

augmented reality, mixed reality, cognitive load, deictic gesture, human-robot interaction

\section{INTRODUCTION}

This paper explores the tradeoffs between different types of mixed reality robotic communication under different levels of user workload.

Successful human-robot interaction in many domains relies on successful communication. Accordingly, there has been a wealth of research on enabling human-robot communication through natural language [30, 51]. However, just like human natural language communication, situated human-robot dialogue is inherently multimodal, and necessarily involves communication channels other than speech. For a host of reasons both egocentric (sensitive only to their own perspective) and allocentric (sensitive to others' perspectives), people regularly use gaze and gesture cues to augment, modify, or replace their natural language utterances. Speakers regularly use deictic gestures such as pointing, for example, to direct interlocutors' attention to objects in the environment, both to reduce the number of words that the speaker must use to refer to their target referents as well as to lower the cognitive burden of listeners in interpreting speakers' utterances.

Due to the near-necessity of deictic gestures in situated communication, human-robot interaction researchers have sought to enable robots to understand [29] and generate [38-40] deictic gestures just as humans do. But while understanding deictic gestures requires only a camera or depth sensor, generation of deictic gestures requires a specific robotic morphology (i.e., expressive robotic arms). This fundamentally limits the gestural capabilities, and thus overall communicative capabilities, of the majority of robotic platforms in use today, such as mobile bases used in warehouses, assistive wheelchairs, and unmanned aerial drones. Moreover, even for robots that do have arms, traditional deictic gestures have fundamental limitations. In contexts such as urban or alpine search and rescue, for example, robots may need to communicate about hardto-describe and/or highly ambiguous referents in novel, uncertain, and unknown environments.

To demonstrate all of these problems, consider an aerial drone in a search and rescue context that needs to generate an utterance such as "I found a victim behind that tree" [cf. 64]. First, the robot is highly unlikely to have an arm mounted to it, and thus physical gesture is simply not a possibility. Second, even if the robot did somehow have an arm mounted on it, a pointing gesture is unlikely to be able to successfully pick out a specific far-off tree, and the natural language needed to disambiguate it is likely to be either extremely complex ("the fourth tree from the left in the clump of trees to the right of the large boulder") or non-human-understandable ("the tree 48.2 meters in that direction").

To address these limitations of traditional "egocentric" physical gestures, researchers have recently been exploring the use of mixed reality deictic gestures [63]: visualizations that can serve the same purpose as traditional deictic gestures, and which fall within the broad category of view-augmenting mixed reality interaction design elements in the Reality-Virtuality Interaction Cube framework of Williams, Szafir, and Chakraborti [60]. Williams et al. [63] divides these new forms of non-egocentric visual gestures into allocentric visualizations that can be displayed in teammates' augmented reality head-mounted displays, and perspective-free visualizations that can be projected onto the ground. Recent work in this space has focused on allocentric gestures such as circles and arrows drawn over target objects [57, 58], as well as ego-sensitive allocentric gestures such as virtual arms [17, 18]. Williams et al. [58], for example [see also 57], demonstrate that (non-ego-sensitive) allocentric virtual gestures, at least when tested in a simulated video-based experiment, have the potential to increase communication accuracy and efficiency, and, when paired with complex referring expressions, are viewed as more effective and likable than purely linguistic communication.

However, to date, mixed reality deictic gestures have only been tested in video-based simulations. In this paper, we present the first demonstration of mixed reality deictic gestures generated on actual 
AR Head-Mounted Displays (the Microsoft Hololens) in the context of task-based human-robot interactions.

Moreover, as previously pointed out by Hirshfield et al. [22], the tradeoffs previously considered by Williams et al. [58] between language and visual gesture may be highly sensitive to the level and type of cognitive load that teammates are under. For example, Hirshfield et al. [22] suggest that it may not be advantageous to rely heavily on visual communication in contexts with high visual load (or to rely heavily on linguistic communication in contexts with high auditory or working memory load). These intuitions are motivated by prior theoretical work on human information processing, including Multiple Resource Theory [56], the Perceptual Load model [27], and the Dual-Target Search model [34].

In this paper we thus also present the first exploration of the tradeoffs between different forms of mixed reality communication in contexts with different types of workload impositions.

The rest of the paper will proceed as follows. In Section 2, we discuss some additional important related work related to both AR-for-HRI and cognitive load estimation. In Section 3, we present the design of a human-subject experiment that uses this technical approach to study the effectiveness of different robot communication styles under different types of cognitive load. In Section 4, we present the results of this experiment. Finally, in Sections 5 and 6 we conclude with a discussion of our results and directions for future work.

\section{RELATED WORK}

\subsection{AR for HRI}

Mixed reality technologies that integrate virtual objects into the physical world have recently sparked research interest in the HumanRobot Interaction (HRI) community [61] because they enable better exchange of information between people and robots, in order to improve shared mental models, calibrated trust, and situation awareness [49].

While there has been significant research on augmented and mixed reality for several decades, $[3,4,7,52,65]$ and acknowledgement of the potential for impact of AR on HRI for many years as well [16, 32], it is only in recent years that there has been significant and sustained interest in AR-for-HRI [61, 62]. Recent works in this area include approaches using AR for robot design [35], calibration [42], and training [46]. Moreover, there are a number of approaches towards communicating robots' perspectives [20], intentions $[2,9,10,12,15]$, and trajectories [14, 31, 36, 54].

One of the best ways to improve human robot interaction is sharing perspectives of human and robot to each other. Amor et al. [1] suggest that projecting human instructions and robot intentions (by highlighting potential target objects) in a constrained and highly structured task environment improves human robot teamwork and produces better task result $[1,2,15]$. Similarly, Sibirtseva et al. [44], present work in which robots receiving natural language instructions reflexively generate augmented reality annotations surrounding candidate referents as they are being disambiguated [44].

Finally, as described above, in our own work, we have investigated the use of AR augmentations as an active rather than passive communication strategy, generated as gestures accompanying natural language communication $[18,57,58]$.

\subsection{Objective Measurements of Cognitive Load}

Hirshfield et al. [22] suggest several contextual factors that may determine when mixed reality deictic gestures are most helpful to human teammates: teammates' cognitive load may dictate whether they are capable of accepting new information; and their auditory and visual perceptual load may dictate the most effective modality to accompany or replace natural language. These neural correlates of cognitive and perceptual states can be collected in real-time using neurological and physiological sensors for unobtrusively measuring humans' brain and physiological data including functional Near-Infrared Spectroscopy (fNIRS), Electroencephalography (EEG), electrodermal activity (EDA), electrocardiogram (ECG), and Respiration sensors [13]. fNIRS, a lightweight and non-invasive device, is gaining popularity in the Human-Computer Interaction community [45], as it offers several advantages over other braincomputer interface (BCI) technologies such as greater spatial resolution, higher signal-to-noise ratio, and better practicality for use in normal working conditions [21, 43], although it is of course subject to other limitations [47, 48], including in HRI contexts [8].

The fNIRS component handles raw data from the sensor and outputs a multilabel vector consisting of three labels (workload, auditory perceptual load, and visual perceptual load) from a multilabel long short-term memory (LSTM) classifier every second. These labels are sent to and processed by the centralized server, which then communicates the appropriate decision to both the robot and the mixed reality headset. In this work we are not yet using the fNIRS component of our architecture, and are instead using experimental manipulation to systematically vary cognitive load.

\section{EXPERIMENT}

In this section we present the design of a human-subject experiment that uses this technical approach to study the effectiveness of different robot communication styles under different types of cognitive load.

\subsection{Hypotheses}

Specifically, this experiment was designed to test the following hypotheses, which formalize the intuitions of Hirshfield et al. [22].

H1 Users under high visual perceptual load will perform quickest when robots rely on complex natural language without the use of mixed reality deictic gestures.

H2 Users under high auditory perceptual load will perform quickest when robots rely on mixed reality deictic gestures without the use of complex natural language.

H3 Users under high working memory load will perform quickest when robots rely on mixed reality deictic gestures without the use of complex natural language.

H4 Users under low overall load will perform quickest when robots rely on mixed reality deictic gestures paired with complex natural language.

\subsection{Task Design}

To assess these hypotheses, we designed a human-subject experiment in which participants interacted with a language-capable robot while wearing the Microsoft HoloLens, over a series of trials, 


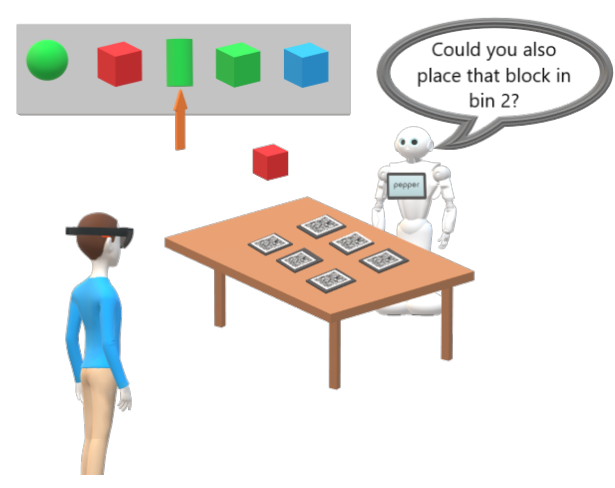

Figure 1: Our experimental setup.

with the robot's communication style and the user's cognitive load systematically varying between trials.

The task used for this experiment employed a dual-task paradigm oriented around a tabletop pick-and-place task. Participants view this task through the Microsoft HoloLens, allowing them to see virtual bins overlaid over a set of fiducial markers on the table, as well as a panel of blocks above the table that changes every few seconds (Fig. 2). As shown in Fig. 1, the Pepper robot is positioned behind the table, ready to interact with the participant.

\section{Primary Task}

The user's primary task is to look out for a particular block in the block panel (selected from among red cube, red sphere, red cylinder, yellow cube, yellow sphere, yellow cylinder, green cube, green sphere, green cylinder ${ }^{1}$ ). These nine blocks were formed by combining three colors red, yellow, green with three shapes cube, sphere, cylinder. Whenever they see this target block, their task is to pick-and-place it into any one of a particular set of bins. For example, a user might be told that whenever they see a red cube they should place it in bins two or three.

Two additional factors increase the complexity of this primary task. First, in order to force participants to remember the full set of candidate bins, rather than just one particular bin from that set, at every point during the task one random bin is marked as unavailable (with the disabled bin changing each time a block is placed in a bin). Second, to allow us to examine auditory load, the user hears a series of syllables playing in the task background (selected from among bah, beh, boh, tah, teh, toh, kah, keh, koh). These nine syllables were formed by combining three consonant sounds b,t,k with three vowel sounds ah,eh,oh. The user is given a target syllable to look out for, and told that whenever they hear this syllable, the bins that they should consider to place blocks in should be exchanged with those they were previously told to avoid. For example, if the user's target bins from among four bins are bins

\footnotetext{
${ }^{1}$ These block colors were chosen for consistent visual processing, as blue is well known to be processed differently within the eye due to spatial and frequency differences of cones between red/green and blue. This did mean that our task was not accessible to red/green colorblind participants, requiring us to remove from our dataset the data of several colorblind participants
}

two and three, and they hear the target syllable, then future blocks will need to be placed instead into bins one and four.

\section{Secondary Task}

Three times per experiment block, the participant encounters a secondary task, in which the Pepper robot interjects and asks the participant to move a particular, currently visible block, to a particular, currently accessible bin.

\subsection{Experimental Design}

We used a Latin square counterbalanced within-subjects experimental design with two independent variables serving as withinsubjects factors:

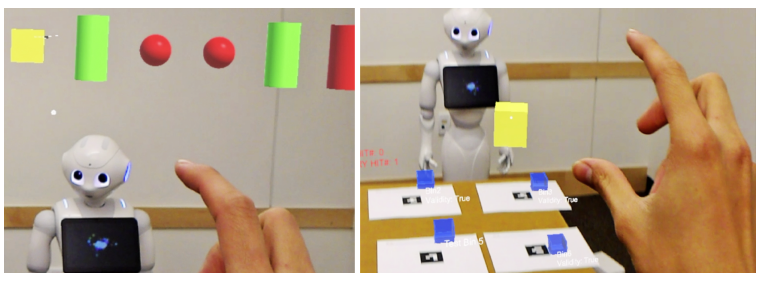

Figure 2: Experiment in progress

\section{Cognitive Load}

Our first independent variable, cognitive load was manipulated through our primary task. Following Beck and Lavie [5], we manipulated communication style by jointly manipulating memory constraints and target/distractor discriminability (cp. [26]), producing four different load profiles: one in which all load was considered low; one in which only working memory load was considered to be high, one in which only visual perceptual load was considered to be high, and one in which only auditory perceptual load was considered to be high.

Working memory load was manipulated as follows: In the high working memory load condition, participants were required to remember the identities of three target bins out of a total of six visible bins, producing a total memory load of seven items when including the two properties of the target block (shape and color) and the two properties of the target syllable (consonant and vowel). In all other conditions, participants were only required to remember the identities of two target bins out of a total of four visible bins, producing a total memory load of six items.

Visual perceptual load was manipulated as follows: In the high visual perceptual load condition, the target block was always difficult to discriminate from distractors due to sharing of one common property with all distractors. For example, if the target block was a red cube, all distractors would be either red or cubes (but not both). In the low visual perceptual load condition, the target block was always easy to discriminate from distractors due to sharing no common properties with any distractors. For example, if the target block was a red cube, no distractors would be red or cubes. 
Auditory perceptual load was manipulated as follows: In the high auditory perceptual load condition, the target syllable was always difficult to discriminate from distractors due to sharing of one common property with all distractors. For example, if the target syllable was $k a h$, all distractors would either start with $k$ or end with ah (but not both). In the low auditory perceptual load condition, the target syllable was always easy to discriminate from distractors due to sharing no common properties with any distractors. For example, if the target syllable was $k a h$, no distractors would either start with $k$ or end with $a h$.

\section{Communication Style}

Our second independent variable, communication style, was manipulated through our secondary task. Following Williams et al. [57] and Williams et al. [58], we manipulated communication style by having the robot exhibit one of three behaviors:

During experiment blocks associated with the complex language communication style condition, the robot with which participants interacted referred to objects using full referring expressions needed to disambiguate those objects.

During experiment blocks associated with the complex language + AR communication style condition, the robot with which participants interacted referred to objects using full referring expressions needed to disambiguate those objects (e.g., "the red sphere"), paired with a mixed reality deictic gesture (an arrow drawn over the object to which the robot was referring).

During experiment blocks associated with the simple language + AR communication style condition, the robot with which participants interacted referred to objects using minimal referring expressions (e.g., "that block"), paired with a mixed reality deictic gesture (an arrow drawn over the object to which the robot was referring).

Following Williams et al. [57] and Williams et al. [58], we did not examine the use of simple language without AR, as that communication style does not always allow complete referent disambiguation, resulting in the user needing to ask for clarification or guess at random between ambiguous options.

\subsection{Measures}

We expected performance improvements to manifest in our experiment in four different ways: task accuracy, task reaction time, perceived mental workload, and perceived communicative effectiveness.

These aspects of performance were measured as follows:

Accuracy was measured for both primary and secondary tasks by logging which virtual object participants clicked on, and determining whether or not this was the object intended by the task or by robot.

Reaction time was measured for both primary and secondary tasks by logging time stamps at the moment participants interacted with virtual objects (both blocks and bins). In the primary task, reaction time was measured as the time between placement of the previous primary target block and picking of the next primary target block. In the secondary task, reaction time was measured as the time between the start of Pepper's utterance and the placement of the secondary target block.
Perceived mental workload was measured using a NASA Task Load Index (NASA TLX) survey[19] administered at the end of each experiment block.

Perceived communicative effectiveness was measured using the modified version of the Gesture Perception Scale [40] previously employed by Williams et al. [57, 58], which was delivered along with the NASA TLX Survey at the end of each experiment block.

\subsection{Procedure}

Upon arriving at the lab, providing informed consent, and completing demographic and visual capability survey, participants were introduced to the task through both verbal instruction and an interactive tutorial.

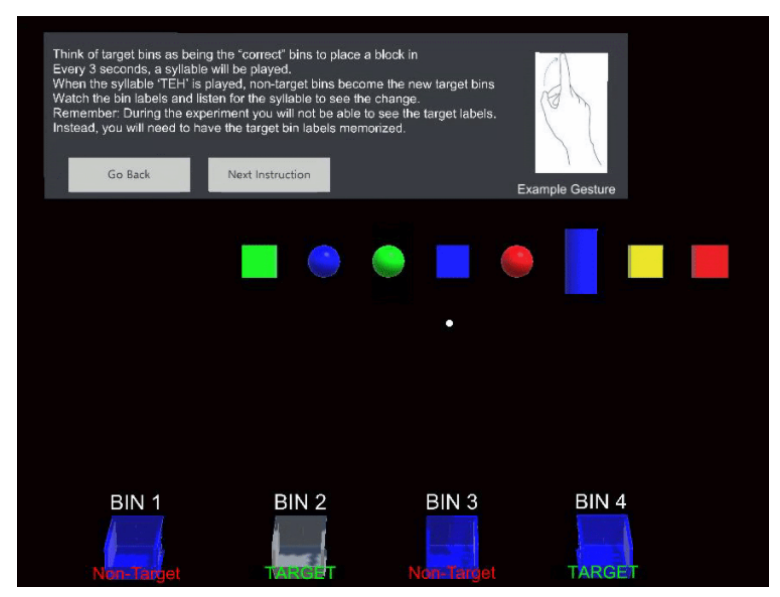

Figure 3: Tutorial

The tutorial scene provides text and visuals that walk the participant through how a round in the experiment will function. When the participant starts the tutorial, they see a panel with text-instructions, a row of blocks, and four bins (Fig. 3). Participants are walked through how to use the HoloLens air tap gesture to pick up blocks and put them in bins through descriptive text and an animation showing an example air tap gesture, and informed of task mechanics with respect to both target/non-target bins and temporarily disabled grey bins. Participants then start to hear syllables being played by the HoloLens. When the target syllable teh plays, the target and non-target bins switch. Each bin on screen is labeled as a 'target' or 'non-target', in order to help the participant understand what is happening when the target syllable plays. These labels are only shown in the tutorial and participants are reminded that they will have to memorize which bins are targets for the actual game. At the end of the tutorial the participant has to successfully put a target block in a target bin three times before they can start the experiment.

After completing this experiment, participants engaged in each of the twelve (Latin square counterbalanced) experiment blocks formed by combining the four cognitive load conditions and the three communication style conditions, with surveys administered after each experiment block. 


\subsection{Participants}

36 participants were recruited from Colorado School of Mines (31 $\mathrm{M}, 5 \mathrm{~F}$ ), ranging in age from 18 to 32 . None had participated in any previous studies from our laboratory.

\subsection{Analysis}

Data analysis was performed within a Bayesian analysis framework using the JASP 0.11.1 [50] software package, using the default settings as justified by Wagenmakers et al. [53]. For each measure, a repeated measures analysis of variance (RM-ANOVA) [11, 33, 37] was performed, using communication style and cognitive load as random factors. Baws factors [28] were then computed for each candidate main effect and interaction, indicating (in the form of a Bayes Factor) for that effect the evidence weight of all candidate models including that effect compared to the evidence weight of all candidate models not including that effect. When sufficient evidence was found in favor of a main effect, the results were further analyzed using a post-hoc Bayesian t-test $[24,55]$ with a default Cauchy prior (center $=0, r=\frac{\sqrt{2}}{2}=0.707$ ). When sufficient evidence was found in favor of an interaction effect, the results were further analyzed using a series of post-hoc paired-samples t-tests each category of cognitive load.

\section{RESULTS}

\subsection{Reaction Time Secondary Task}

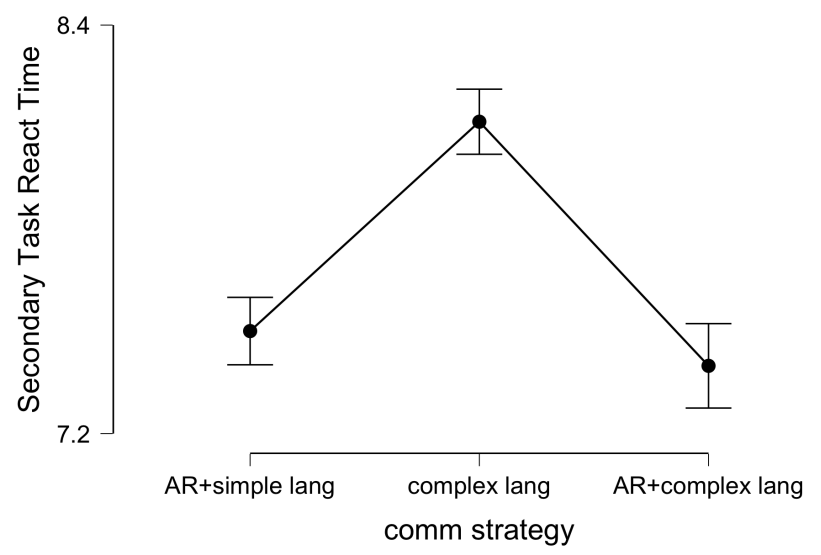

Figure 4: Effect of communication strategy (complex language + AR vs. complex language vs. simple language + AR) on secondary task reaction time.

Our results provided extreme evidence in favor of effects of both communication style (Bf 3.109e29) ${ }^{2}$ and cognitive load (Bf 9.881e9) on secondary task reaction time, as shown in Figs. 4 and 5, as well as an interaction between communication style and cognitive load

\footnotetext{
${ }^{2}$ Bayes Factors above 100 indicate extreme evidence in favor of a hypothesis [6, 23] Here, for example, our Baws Factor Bf of 7.024e25 suggests that our data were 7.024e25 times more likely to be generated under models in which communication style is included than under those in which it is not.
}

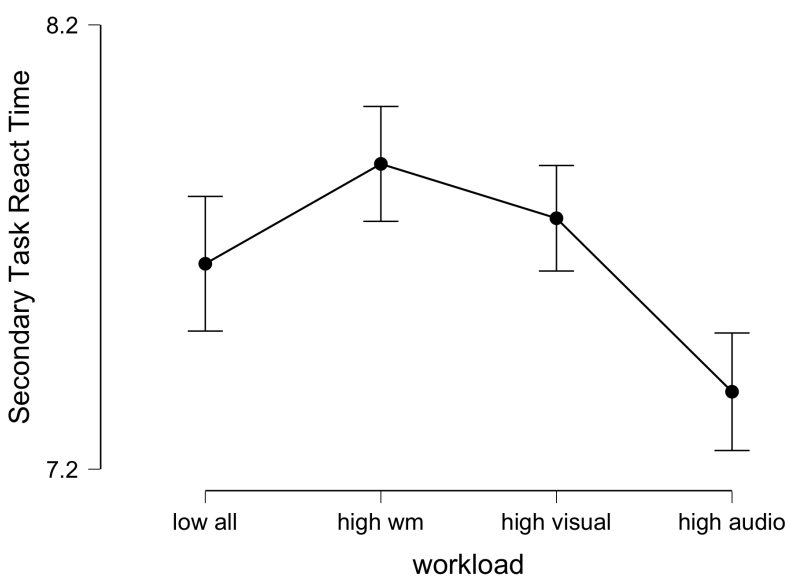

Figure 5: Effect of workload (Low All) vs. (High Visual) vs. (High Auditory) vs. (High Working Memory) on participant's secondary task reaction time.

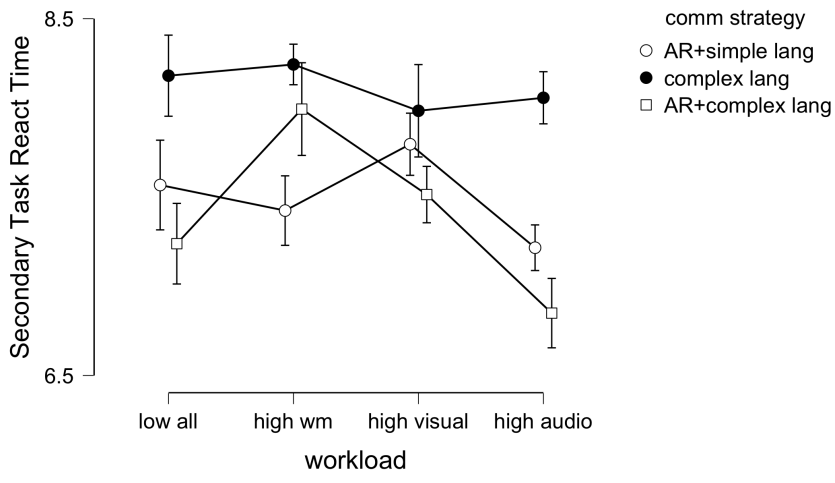

Figure 6: Effect of both workload and communication strategy on participant's secondary task reaction time.

(Bf. 1.160e12) on reaction time, as shown in Fig. 6.

Post-hoc analysis of the main effect of communication style on secondary task reaction time revealed significant differences specifically between the use of complex language alone ( $\mu=8.116 \mathrm{sec})$ and both complex language $+\mathrm{AR}(\mu=7.399 \mathrm{sec}, \mathrm{Bf} 2.955 \mathrm{e} 21)$ and simple language + AR ( $\mu=7.501 \mathrm{sec}$, Bf 9.396e15), with anecdotal evidence against a difference between complex language $+\mathrm{AR}$ and complex language alone $(\mathrm{Bf}=.46$ in favor of an effect; $1 / .46=\mathrm{Bf}$ 2.14 against an effect)

This yields a preference ordering where complex language $<$ (simple language $+A R=$ complex language $+A R)$ when cognitive load is not considered.

Post-hoc analysis of the main effect of cognitive load on secondary task reaction time revealed significant differences specifically between conditions with high auditory perceptual load ( $\mu=$ $7.374 \mathrm{sec}, \sigma=0.454 \mathrm{sec}$ ) and all other conditions, i.e., low overall load ( $\mu=7.662 \mathrm{sec}, \sigma=0.684 \mathrm{sec}$, Bf 2931.437), high visual perceptual 
load ( $\mu=7.765 \mathrm{sec}, \sigma=0.574 \mathrm{sec}$, Bf 283407.874), and high working memory load ( $\mu=7.887 \mathrm{sec}, \sigma=0.551 \mathrm{sec}$, Bf 1.343e9), as well as between conditions with high working memory load and those with low overall load (Bf 13.381).

This yields a preference ordering where high auditory perceptual load < ( (low overall load $<$ high working memory load $)$ = high visual perceptual load) when communication style is not considered.

Post-hoc analysis of the interaction effect between communication style and cognitive load on secondary task reaction revealed the following additional findings:

Low Overall Load Extreme evidence was found under low overall load between each pair of communication strategies: simple language $+\mathrm{AR}(\mu=7.568 \mathrm{sec}, \sigma=0.732 \mathrm{sec})$ vs complex language alone $(\mu=8.195 \mathrm{sec}, \sigma=0.685 \mathrm{sec}$, Bf 8.995e6); simple language + AR vs complex language $+\mathrm{AR}(\mu=7.253 \mathrm{sec}, \sigma=0.654 \mathrm{sec}$, Bf 703110.101) complex language alone vs complex language + AR Bf 1.281e13.

This yields a preference ordering where complex language alone $<$ simple language $+A R<$ complex language $+A R$ in the low overall load condition.

High Working Memory Load Extreme evidence was found under high working memory load between simple language $+\mathrm{AR}$ ( $\mu=7.439 \mathrm{sec}, \sigma=0.565 \mathrm{sec})$ and both complex language alone ( $\mu=8.240 \mathrm{sec}, \sigma=0.327 \mathrm{sec}, \mathrm{Bf} 1.080 \mathrm{e} 7)$ and complex language $+\mathrm{AR}$ $(\mu=7.988 \mathrm{sec}, \sigma=0.746 \mathrm{sec}$, Bf 2076.594).

This yields a preference ordering where (complex language alone $=$ complex language $+A R)<$ simple language $+A R$ in the high working memory load condition.

High Visual Perceptual Load Moderate to extreme evidence was found under high visual perceptual load between complex language $+\mathrm{AR}(\mu=7.506 \mathrm{sec}, \sigma=0.456 \mathrm{sec})$ and both complex language alone $(\mu=7.997, \sigma=0.747 \mathrm{sec}, \mathrm{Bf} 1449.784)$ and simple language $+\mathrm{AR}$ $(\mu=7.781 \mathrm{sec}, \sigma=0.508 \mathrm{sec}$, Bf 5.336).

This yields a preference ordering where (simple language $+A R=$ complex language alone $)<$ complex language $+A R$ in the high visual perceptual load condition.

High Auditory Perceptual Load Extreme evidence was found under high auditory perceptual load between each pair of communication strategies (simple language $+\mathrm{AR}(\mu=7.219 \mathrm{sec}, \sigma=0.367 \mathrm{sec})$ vs complex language alone ( $\mu=8.050 \mathrm{sec}, \sigma=0.421$, Bf 7.374e6); simple language $+\mathrm{AR}$ vs complex language $+\mathrm{AR}(\mu=6.859 \mathrm{sec}$, $\sigma=0.560 \mathrm{sec}$, Bf 35.760); complex language alone vs complex language + AR (Bf 1.126e13).

This yields a preference ordering where complex language alone $<$ simple language $+A R<$ complex language $+A R$ in the high auditory perceptual load condition.

\section{Primary Task}

Strong evidence was found against any effects of communication style or cognitive load on primary task reaction time (All Bfs $>20$ against an effect).

\subsection{Accuracy}

Strong evidence was found against any effects of communication style or cognitive load on primary or secondary task accuracy (All Bfs $>27$ against an effect).

\subsection{Perceived Mental Workload}

Anecdotal to strong evidence was found against any effects of communication style or cognitive load on perceived mental workload (Bfs between 22.43 and 40.91 against an effect).

\subsection{Perceived Communicative Effectiveness}

Anecdotal to strong evidence was found against any effects of communication style or cognitive load on perceived communicative effectiveness (Bfs between 2.23 and 83.33 against an effect on all questions).

\section{DISCUSSION}

Our results suggest that although humans may not be aware of differences in their performance or mental workload when different mixed reality robotic communication styles are used, or when they are under different types of cognitive load, both of these factors do in fact influence the speed at which they are able to accomplish tasks.

First, our results suggest that different types of mental workload do, unsurprisingly, impact task task, with participants under low overall load reacting more quickly than participants under high working memory load. What is surprising is that participants under high auditory load clearly demonstrated the fastest reaction times overall. It is not yet clear how to interpret this result, but it is possible that this effect is due to individuals generally responding faster to auditory stimuli that visual [25].

Second, our results suggest, unsurprisingly, that different communication strategies impact task time. In fact, our results exactly match what we observed in previous experiments [58]: participants demonstrate slower reaction times when complex language alone is used, with no clear differences between simple and complex language when it is augmented with a mixed reality deictic gesture.

Finally, our results suggest a complex interplay between communication style and cognitive load. Specifically, our results suggest that while using complex language + AR resulted in the best task time in most workload conditions (an encouraging result given that our previous work has shown that participants find robots most likeable when they use this communication style), this does not hold true when users are under high working memory load. Rather, when users are under high working memory load, it is best to use simple language $+\mathrm{AR}$, to avoid overloading participants.

Overall, these results support hypotheses $\mathrm{H} 3$ and $\mathrm{H} 4$, but fail to support hypotheses $\mathrm{H} 1$ and $\mathrm{H} 2$. While our original expectation was that the differences between communication styles under different cognitive load profiles would primarily be grounded in whether communication style was overall visual or overall visual, in fact 


\section{C8: LOW WorkingMem, HIGH Vis, LOW Au | Robot Strategy: Complex Language Only}
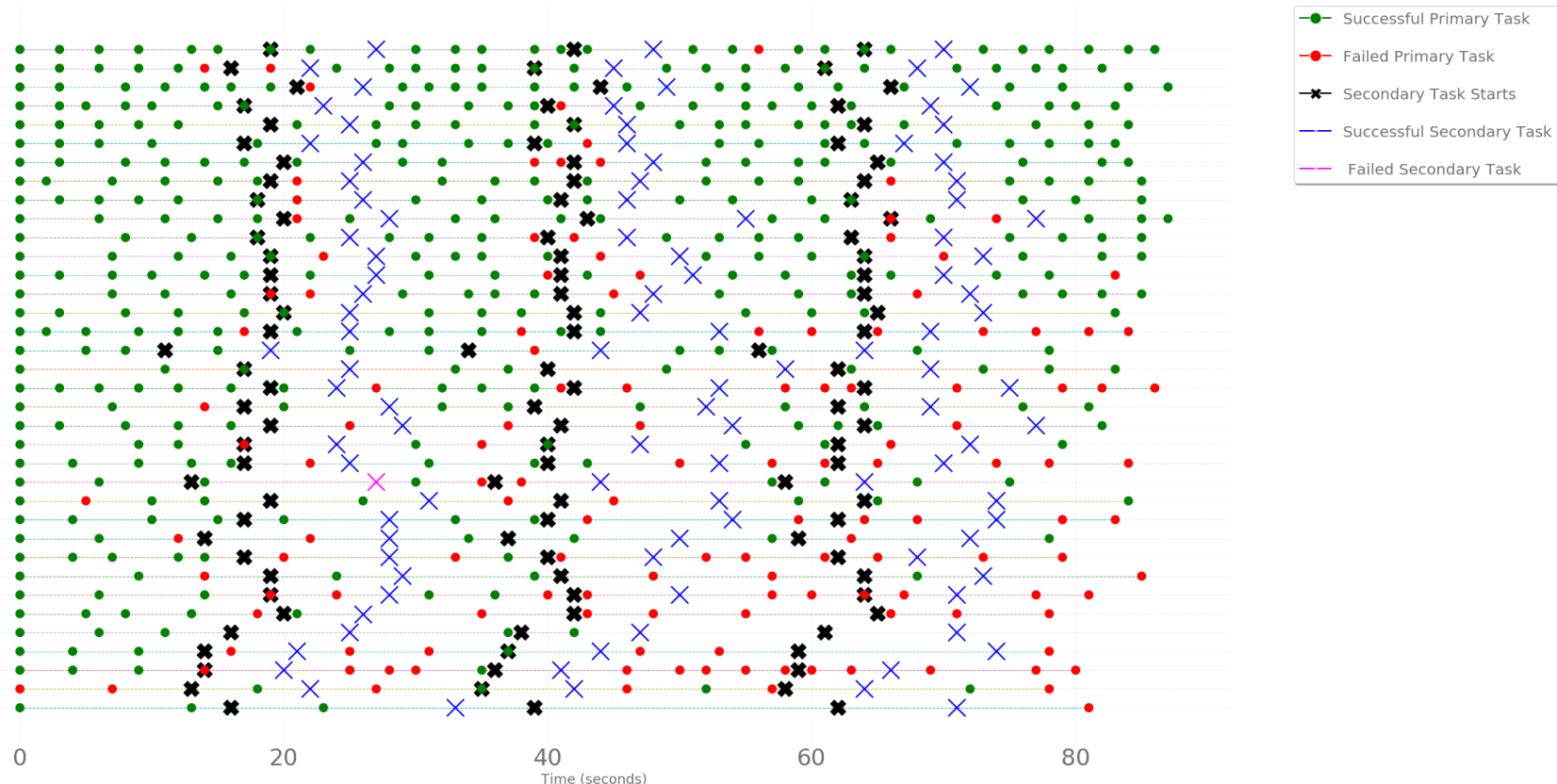

Figure 7: Visualization of participant performance in the Complex Language Only / High visual perceptual load condition

what we observed is that visual augmentations are always helpful, and differences in effectiveness between workload conditions depend entirely on whether or not the user is under high cognitive load.

While we observed clear impacts of workload profiles on task time, participants did not demonstrate any differences in perceived workload or perceived effectiveness. This could be the case that the differences in reaction time simply were not large enough for participants to notice: the observed differences were on the order of one second of reaction time when overall reaction time was around 7.5 seconds. Participants may simply not have noticed a $15 \%$ speed increase in certain conditions, or may not have attributed it to the robot.

This could also be the case due to overall task difficulty. While participants' TLX scores had a mean value of approximately 21 out of 42 points in all conditions (i.e., the data was nearly perfectly centered around "medium" load), analysis of individual performance trajectories demonstrates that the task was sufficiently difficult that many participants experienced catastrophic primary task shedding, often immediately after a primary task (likely due to missing an auditory cue while dealing with a secondary task). As illustrated in Fig. $7^{3}$, task time and accuracy varied significantly between participants. In this figure the dark X markers represent the time the robot started uttering a secondary task requests. As can be seen, most participants performed well on the primary task (resulting in many green dots) up until immediately after the first or second secondary task request. As can also be seen, when participants made

\footnotetext{
${ }^{3}$ This figure shows only one condition, the complex language/High visual load condition), for the sake of space. All twelve condition plots, however, show similar results to what we observed here.
}

a mistake, except in cases where the error fell between secondary task initiation and completion, they often failed to recover from the failure.

\section{CONCLUSION}

The ultimate goal of our research is to enable adaptive mixed reality communication for human-robot interaction. In this paper, we presented the first experimental steps towards achieving this goal. Our results provide critical insights for the future design of our proposed adaptive system.

In future work, we plan to complete our integration of the fNIRS neurophysiological sensor with the current mixed reality robotic architecture, in order to accurately measure changes in mental workload within experimental conditions, as well as in task contexts that do not have tightly controlled levels of workload. We further plan to integrate all three components together with the Distributed Integrated Affect Reflection and Cognition (DIARC) architecture to leverage its rich natural language understanding and generation capabilities [41,59].

Finally, in future work we also hope to consider how robots can tailor gestural cues to be easily discriminable from both background visual stimuli and other task targets without placing the human teammate at risk of inattentional blindness.

\section{ACKNOWLEDGEMENT}

This research was funded in part by NSF grants IIS-1909864 and CNS-1810631. 


\section{REFERENCES}

[1] Heni Ben Amor, Ramsundar Kalpagam Ganesan, Yash Rathore, and Heather Ross. 2018. Intention projection for human-robot collaboration with mixed reality cues. In Proceedings of the 1st International Workshop on Virtual, Augmented, and Mixed Reality for HRI (VAM-HRI).

[2] Rasmus S Andersen, Ole Madsen, Thomas B Moeslund, and Heni Ben Amor. 2016. Projecting robot intentions into human environments. In 2016 25th IEEE International Symposium on Robot and Human Interactive Communication (ROMAN). IEEE, 294-301.

[3] Ronald Azuma. 1997. A Survey of Augmented Reality. Presence: Teleoperators \& Virtual Environments 6 (1997), 355-385.

[4] Ronald Azuma, Yohan Baillot, Reinhold Behringer, Steven Feiner, Simon Julier and Blair MacIntyre. 2001. Recent advances in augmented reality. IEEE computer graphics and applications 21, 6 (2001), 34-47.

[5] Diane M Beck and Nilli Lavie. 2005. Look here but ignore what you see: effects of distractors at fixation. Journal of Experimental Psychology: Human Perception and Performance 31, 3 (2005), 592.

[6] James O Berger and Luis R Pericchi. 1996. The intrinsic Bayes factor for model selection and prediction. f. Amer. Statist. Assoc. 91, 433 (1996), 109-122.

[7] Mark Billinghurst, Adrian Clark, Gun Lee, et al. 2015. A survey of augmented reality. Foundations and Trends® in Human-Computer Interaction 8, 2-3 (2015), 73-272.

[8] Cody Canning and Matthias Scheutz. 2013. Functional near-infrared spectroscopy in human-robot interaction. Fournal of Human-Robot Interaction 2, 3 (2013), 62 84.

[9] Tathagata Chakraborti, Sarath Sreedharan, Anagha Kulkarni, and Subbarao Kambhampati. 2017. Alternative modes of interaction in proximal human-in-the-loop operation of robots. arXiv preprint arXiv:1703.08930 (2017).

[10] Mark Cheli, Jivko Sinapov, Ethan E Danahy, and Chris Rogers. 2018. Towards an augmented reality framework for k-12 robotics education. In Proceedings of the 1st International Workshop on Virtual, Augmented, and Mixed Reality for HRI (VAM-HRI).

[11] Martin J Crowder. 2017. Analysis of repeated measures. Routledge.

[12] Andrew Dudley, Tathagata Chakraborti, and Subbarao Kambhampati. 2018. v2v Communication for Augmenting Reality Enabled Smart HUDs to Increase Situational Awareness of Drivers. (2018).

[13] S. Fairclough. 2009. Fundamentals of physiological computing. Interacting with Computers 21 (2009), 133-145.

[14] Jared A Frank, Matthew Moorhead, and Vikram Kapila. 2017. Mobile Mixedreality interfaces That enhance human-robot interaction in shared spaces. Frontiers in Robotics and AI 4 (2017), 20.

[15] Ramsundar Kalpagam Ganesan, Yash K Rathore, Heather M Ross, and Heni Ben Amor. 2018. Better teaming through visual cues: how projecting imagery in a workspace can improve human-robot collaboration. IEEE Robotics \& Automation Magazine 25, 2 (2018), 59-71.

[16] Scott A Green, Mark Billinghurst, XiaoQi Chen, and J Geoffrey Chase. 2008 Human-robot collaboration: A literature review and augmented reality approach in design. International journal of advanced robotic systems 5, 1 (2008), 1.

[17] Thomas Groechel, Zhonghao Shi, Roxanna Pakkar, and Maja J Matarić. 2019. Using Socially Expressive Mixed Reality Arms for Enhancing Low-Expressivity Robots. In 2019 28th IEEE International Conference on Robot and Human Interactive Communication (RO-MAN). IEEE, 1-8.

[18] Jared Hamilton, Nhan Tran, and Tom Williams. 2020. Tradeoffs Between Effectiveness and Social Perception When Using Mixed Reality to Supplement Gesturally Limited Robots. In Proceedings of the 3rd International Workshop on Virtual, Augmented, and Mixed Reality for HRI

[19] S.G. Hart and L.E. Staveland. 1988. Development of NASA-TLX (Task Load Index): Results of empirical and theorical research. Amsterdam, pp $139-183$.

[20] Hooman Hedayati, Michael Walker, and Daniel Szafir. 2018. Improving collocated robot teleoperation with augmented reality. In Proceedings of the 2018 ACM/IEEE International Conference on Human-Robot Interaction. 78-86.

[21] Hirshfield., R. Gulotta, S. Hirshfield, S. Hincks, M. Russell, T. Williams, and R Jacob. [n.d.]. This is your brain on interfaces: enhancing usability testing with functional near infrared spectroscopy. In SIGCHI. ACM.

[22] Leanne Hirshfield, Tom Williams, Natalie Sommer, Trevor Grant, and Senem Velipasalar Gursoy. 2018. Workload-driven modulation of mixed-reality robot-human communication. In Proceedings of the Workshop on Modeling Cognitive Processes from Multimodal Data. ACM, 3.

[23] Andrew F Jarosz and Jennifer Wiley. 2014. What are the odds? A practical guide to computing and reporting Bayes factors. The fournal of Problem Solving 7, 1 (2014), 2.

[24] Harold Jeffreys. 1938. Significance tests when several degrees of freedom arise simultaneously. Proc. Royal Society of London. Series A, Math. and Phys. Sci. (1938).

[25] Shelton Jose and Kumar Gideon Praveen. 2010. Comparison between auditory and visual simple reaction times. Neuroscience \& Medicine 2010 (2010).

[26] Nilli Lavie. 1995. Perceptual load as a necessary condition for selective attention. fournal of Experimental Psychology: Human perception and performance 21, 3
(1995), 451

[27] Nilli Lavie. 2006. The role of perceptual load in visual awareness. Brain research 1080, 1 (2006), 91-100.

[28] S. Mathôt. 2017. Bayes like a Baws: Interpreting Bayesian repeated measures in JASP [Blog Post]. https://www.cogsci.nl/blog/interpreting-bayesian-repeatedmeasures-in-jasp.

[29] Cynthia Matuszek, Liefeng Bo, Luke Zettlemoyer, and Dieter Fox. 2014. Learning from unscripted deictic gesture and language for human-robot interactions. In Twenty-Eighth AAAI Conference on Artificial Intelligence.

[30] Nikolaos Mavridis. 2015. A review of verbal and non-verbal human-robot interactive communication. Robotics and Autonomous Systems 63 (2015), 22-35.

[31] Sebastian Meyer zu Borgsen, Patrick Renner, Florian Lier, Thies Pfeiffer, and Sven Wachsmuth. 2018. Improving human-robot handover research by mixed reality techniques. In VAM-HRI 2018. The Inaugural International Workshop on Virtual, Augmented and Mixed Reality for Human-Robot Interaction. Proceedings.

[32] Paul Milgram, Shumin Zhai, David Drascic, and Julius Grodski. 1993. Applications of augmented reality for human-robot communication. In Proceedings of 1993 IEEE/RSF International Conference on Intelligent Robots and Systems (IROS'93), Vol. 3. IEEE, 1467-1472.

[33] RD Morey and JN Rouder. 2014. BayesFactor (Version 0.9. 9).

[34] Alex Muhl-Richardson, Katherine Cornes, Hayward J Godwin, Matthew Garner, Julie A Hadwin, Simon P Liversedge, and Nick Donnelly. 2018. Searching for two categories of target in dynamic visual displays impairs monitoring ability. Applied cognitive psychology 32, 4 (2018), 440-449.

[35] Christopher Peters, Fangkai Yang, Himangshu Saikia, Chengjie Li, and Gabriel Skantze. 2018. Towards the use of mixed reality for hri design via virtual robots. In Proceedings of the 1st International Workshop on Virtual, Augmented, and Mixed Reality for HRI (VAM-HRI).

[36] Eric Rosen, David Whitney, Elizabeth Phillips, Gary Chien, James Tompkin, George Konidaris, and Stefanie Tellex. 2020. Communicating robot arm motion intent through mixed reality head-mounted displays. In Robotics Research. Springer, 301-316.

[37] Jeffrey N Rouder, Richard D Morey, Paul L Speckman, and Jordan M Province. 2012. Default Bayes factors for ANOVA designs. Fournal of Mathematical Psychology 56, 5 (2012), 356-374.

[38] Maha Salem, Friederike Eyssel, Katharina Rohlfing, Stefan Kopp, and Frank Joublin. 2013. To err is human (-like): Effects of robot gesture on perceived anthropomorphism and likability. International fournal of Social Robotics 5, 3 (2013), 313-323.

[39] Maha Salem, Stefan Kopp, Ipke Wachsmuth, Katharina Rohlfing, and Frank Joublin. 2012. Generation and evaluation of communicative robot gesture. International fournal of Social Robotics 4, 2 (2012), 201-217.

[40] Allison Sauppé and Bilge Mutlu. 2014. Robot deictics: How gesture and context shape referential communication. In 2014 9th ACM/IEEE International Conference on Human-Robot Interaction (HRI). IEEE, 342-349.

[41] Matthias Scheutz, Thomas Williams, Evan Krause, Bradley Oosterveld, Vasanth Sarathy, and Tyler Frasca. 2019. An overview of the distributed integrated cognition affect and reflection diarc architecture. In Cognitive Architectures. Springer, 165-193.

[42] Manfred Schönheits and Florian Krebs. 2018. Embedding ar in industrial hri applications. In Proceedings of the 1st International Workshop on Virtual, Augmented, and Mixed Reality for HRI (VAM-HRI).

[43] Abdul Serwadda, Vir V Phoha, Sujit Poudel, Leanne M Hirshfield, Danushka Bandara, Sarah E Bratt, and Mark R Costa. 2015. ANIRS: A new modality for brain activity-based biometric authentication. In 2015 IEEE 7th International Conference on Biometrics Theory, Applications and Systems (BTAS). IEEE, 1-7.

[44] Elena Sibirtseva, Dimosthenis Kontogiorgos, Olov Nykvist, Hakan Karaoguz, Iolanda Leite, Joakim Gustafson, and Danica Kragic. 2018. A comparison of visualisation methods for disambiguating verbal requests in human-robot interaction. In 2018 27th IEEE International Symposium on Robot and Human Interactive Communication (RO-MAN). IEEE, 43-50.

[45] Erin Treacy Solovey, Audrey Girouard, Krysta Chauncey, Leanne M Hirshfield, Angelo Sassaroli, Feng Zheng, Sergio Fantini, and Robert JK Jacob. 2009. Using fNIRS brain sensing in realistic HCI settings: experiments and guidelines. In Proceedings of the 22nd annual ACM symposium on User interface software and technology. ACM, 157-166.

[46] Daniele Sportillo, Alexis Paljic, Luciano Ojeda, Giacomo Partipilo, Philippe Fuchs, and Vincent Roussarie. 2018. Learn how to operate semi-autonomous vehicles with Extended Reality.

[47] Megan Strait, C Canning, and M Scheutz. 2013. Limitations of NIRS-based BCI for realistic applications in human-computer interaction. In BCI Meeting. 6-7.

[48] Megan Strait and Matthias Scheutz. 2014. What we can and cannot (yet) do with functional near infrared spectroscopy. Frontiers in neuroscience 8 (2014), 117

[49] Daniel Szafir. 2019. Mediating Human-Robot Interactions with Virtual, Augmented, and Mixed Reality. In International Conference on Human-Computer Interaction. Springer, 124-149.

[50] JASP Team. 2018. JASP (Version 0.8.5.1)[Computer software]. 
[51] Stefanie Tellex, Nakul Gopalan, Hadas Kress-Gazit, and Cynthia Matuszek. 2020 Robots That Use Language. Annual Review of Control, Robotics, and Autonomous Systems 3 (2020).

[52] DWF Van Krevelen and Ronald Poelman. 2010. A survey of augmented reality technologies, applications and limitations. International journal of virtual reality 9, 2 (2010), 1-20.

[53] EJ Wagenmakers, J Love, M Marsman, T Jamil, A Ly, and J Verhagen. 2018 Bayesian inference for psychology, Part II: Example applications with JASP. Psychonomic Bulletin and Review 25, 1 (2018), 35-57.

[54] Michael Walker, Hooman Hedayati, Jennifer Lee, and Daniel Szafir. 2018. Communicating robot motion intent with augmented reality. In Proceedings of the 2018 ACM/IEEE International Conference on Human-Robot Interaction. 316-324.

[55] Peter H Westfall, Wesley O Johnson, and Jessica M Utts. 1997. A Bayesian perspective on the Bonferroni adjustment. Biometrika 84, 2 (1997), 419-427.

[56] Christopher D Wickens. 2002. Multiple resources and performance prediction. Theoretical issues in ergonomics science 3, 2 (2002), 159-177.

[57] Tom Williams, Matthew Bussing, Sebastian Cabrol, Elizabeth Boyle, and Nhan Tran. 2019. Mixed Reality Deictic Gesture for Multi-Modal Robot Communication In Proceedings of the 14th ACM/IEEE International Conference on Human-Robot Interaction.

[58] Tom Williams, Matthew Bussing, Sebastian Cabrol, Ian Lau, Elizabeth Boyle, and Nhan Tran. 2019. Investigating the Potential Effectiveness of Allocentric Mixed Reality Deictic Gesture. In Proceedings of the 11th International Conference on Virtual, Augmented, and Mixed Reality.
[59] Tom Williams and Matthias Scheutz. 2017. Referring Expression Generation Under Uncertainty: Algorithm and Evaluation Framework. In Proceedings of the 10th International Conference on Natural Language Generation.

[60] Tom Williams, Daniel Szafir, and Tathagata Chakraborti. 2019. The RealityVirtuality Interaction Cube. In Proceedings of the 2nd International Workshop on Virtual, Augmented, and Mixed Reality for HRI.

[61] Tom Williams, Daniel Szafir, Tathagata Chakraborti, and Heni Ben Amor. 2018. Virtual, augmented, and mixed reality for human-robot interaction. In Companion of the 2018 ACM/IEEE International Conference on Human-Robot Interaction. ACM, 403-404.

[62] Tom Williams, Daniel Szafir, Tathagata Chakraborti, and Elizabeth Phillips. 2019. Virtual, augmented, and mixed reality for human-robot interaction (vam-hri). In 2019 14th ACM/IEEE International Conference on Human-Robot Interaction (HRI). IEEE, 671-672.

[63] Tom Williams, Nhan Tran, Josh Rands, and Neil T Dantam. 2018. Augmented, mixed, and virtual reality enabling of robot deixis. In International Conference on Virtual, Augmented and Mixed Reality. Springer, 257-275.

[64] Tom Williams, Fereshta Yazdani, Prasanth Suresh, Matthias Scheutz, and Michae Beetz. 2019. Dempster-shafer theoretic resolution of referential ambiguity. $A u$ tonomous Robots 43, 2 (2019), 389-414.

[65] Feng Zhou, Henry Been-Lirn Duh, and Mark Billinghurst. 2008. Trends in augmented reality tracking, interaction and display: A review of ten years of ISMAR. In 2008 7th IEEE/ACM International Symposium on Mixed and Augmented Reality. IEEE, 193-202. 